\title{
Endoplasmic reticulum stress induced by lipopolysaccharide is involved in the association between inflammation and autophagy in INS-1 cells
}

\author{
HAN LIU, JIA-JING YIN, MING-MING CAO, GUO-DONG LIU, YING SU and YAN-BO LI
}

Department of Endocrinology, First Affiliated Hospital of Harbin Medical University, Harbin, Heilongjiang 150001, P.R. China

Received October 18, 2016; Accepted June 13, 2017

DOI: $10.3892 / \mathrm{mmr} .2017 .7350$

\begin{abstract}
Type 2 diabetes is a chronic inflammatory disease. Autophagy, the dynamic process of lysosomal degradation of damaged organelles and proteins, may protect $\beta$-cells from destruction by inflammation in type 2 diabetes. The present study investigated the role of autophagy, inflammation and endoplasmic reticulum (ER) stress in type 2 diabetes. INS-1 cells were incubated with lipopolysaccharide. The chemical chaperone 4-phenylbutyric acid was used to inhibit ER stress, and 3-methyadenine (3-MA) was used to inhibit autophagy. Apoptosis was detected by flow cytometry and cell proliferation using Cell Counting kit-8 solution. Light chain-3B, interleukin (IL) $1 \beta$, caspase- 1 and $\mathrm{C} / \mathrm{EBP}$ homologous protein production were assessed by western blotting, and rat activating transcription factor 4 and rat binding immunoglobulin heavy chain protein gene expression were determined by real-time reverse transcription-polymerase chain reaction. The results showed that inhibiting autophagy with 3-MA unexpectedly contributed to cell death in $\beta$-cells. This response was associated with an increase in inflammatory cytokines, including IL1 $\beta$ and caspase-1. Inhibiting ER stress with 4-phenylbutyric acid led to a decrease in cell apoptosis. These results showed that autophagy may have a protective effect by reducing inflammatory cytokines in $\beta$-cells. In addition, the inositol-requiring enzyme 1 pathway mediated the ER stress associated with autophagy and inflammatory cytokines (IL1 $\beta$ and caspase-1). Therefore, inflammatory cytokines may be critical signalling nodes, which are associated with ER stress-mediated $\beta$-cell death.
\end{abstract}

Correspondence to: Dr Yan-Bo Li, Department of Endocrinology, First Affiliated Hospital of Harbin Medical University, 23 You Zheng Street, Harbin, Heilongjiang 150001, P.R. China

E-mail: liyanbo2016@163.com

Abbreviations: 3-MA, 3-methyadenine; ER, endoplasmic reticulum; PBA, 4-phenylbutyric acid; NLRP, NOD-like receptor; LC3, light chain 3; LPS, lipopolysaccharide; CCK8, Cell Counting kit-8; AV, autophagic vacuoles; AL, autophagolysosomes

Key words: type 2 diabetes, autophagy, inflammatory cytokines, endoplasmic reticulum stress, lipopolysaccharide

\section{Introduction}

Inflammation has been implicated in the pathophysiology of type 2 diabetes. During insulitis, activated macrophages and T-cells release cytokines, including interleukin (IL) $1 \beta$, tumor necrosis factor (TNF)- $\alpha$ and interferon (IFN)- $\gamma$, close to $\beta$-cells, contributing to $\beta$-cell dysfunction and death $(1,2)$. Additionally, there is evidence that elevated levels of IL1 $\beta$, IL6, monocyte chemotactic protein 1 and C-reactive protein are predictive of type 2 diabetes (3). Previous findings suggest that islet $\beta$-cells secrete cytokines by themselves, which induce inflammatory responses. In this process, thioredoxin-interacting protein (TXNIP) is induced by endoplasmic reticulum (ER) stress through the protein kinase RNA-like endoplasmic reticulum kinase (PERK) and inositol-requiring enzyme 1 (IRE1) pathways, which activates the production of IL1 $\beta$ by the NOD-like receptor (NLRP) 3/caspase1 inflammasome, and mediates ER stress-mediated $\beta$-cell death (4).

Autophagy is a major pathway for the delivery of proteins, organelles, lipids, DNA and RNA to lysosomes, where they are to be degraded and recycled in the vacuole. Autophagy provides pools of raw material for anabolic processes and drives a continuous flow of materials in a degradation-regeneration cycle within the cell (5). Paradoxically, autophagy is considered to be a cell-fate decision maker and may lead to a form of non-apoptotic cell death, which is termed type 2 programmed cell death (6). Therefore, whether autophagy protects cells from diverse types of injuries or promotes cell death may depend on either the cellular or the environmental context.

At present, it is known that autophagy is induced by ER stress in mammalian cells and in plants $(7,8)$. Although the molecular mechanism linking ER stress and autophagy remains to be elucidated, the process of cleavage and lipidation of microtubule-associated protein 1 light chain 3 (LC3) into LC3-II is reportedly mediated by the phosphorylation of PERK/eukaryotic translation initiation factor 2A (eIF2a) (6). No autophagosomes were observed in an ire $1 \beta$-knockout mutant when treated with ER stress agents, suggesting that the ER stress sensor, IRE1 $\beta$ b is required for ER stress-induced autophagy $(8,9)$.

All of the above factors may be involved in diabetes. In the present study, the potential involvement of autophagy in the inflammatory response was investigated in INS-1 cells. 
In addition, whether IRE-1-pathway-mediated ER stress is involved in the associated between inflammation and autophagy in diabetes was examined.

\section{Materials and methods}

Cell culture. INS-1 cells from rats (cells were provided by the Department of Endocrinology, The Second Affiliated Hospital of Harbin Medical University, Heilongjiang, China) were passaged in RPMI-1640 medium (Hyclone; GE Healthcare Life Sciences, USA), supplemented with $10 \%$ (v/v) fetal bovine serum (FBS; Sijiqing, Hangzhou, China), in a humidified atmosphere containing $95 \%$ air and $5 \% \mathrm{CO}_{2}(10)$. The cells were treated with $100 \mathrm{ng} / \mathrm{ml}$ lipopolysaccharide (LPS) to activate ER stress-induced inflammatory cytokines, including IL1 $\beta$ and caspase-1. 4-phenylbutyric acid (PBA; $2.5 \mathrm{mmol} / \mathrm{l}$; pH 7.4; Sigma; Merck Millipore, Darmstadt, Germany) was used to inhibit the IRE-1 pathway and mediate ER stress, and 3-methyadenine (3-MA; 5 mmol/1; Sigma; Merck Millipore) was used to inhibit autophagy $(10,11)$.

Cell Counting kit-8 (CCK8) viability assay. The viability of cells was assessed using CCK8 (Dojindo Laboratories, Kumamoto, Japan) according to the manufacturer's protocol. CCK8 is more sensitive than 3-(4,5-dimethylthiazol-2-yl)-2, 5 -diphenyltetrazolium bromide assay (10). The INS-1 cells were plated in 96 -well plates at a density of $5 \times 10^{7} 1^{-1}$ and treated with $10 \mathrm{ml} \mathrm{CCK} 8$ at $37^{\circ} \mathrm{C}$ for $1 \mathrm{~h}$. Absorbance was measured at $450 \mathrm{~nm}$ using a microplate reader (10).

Annexin V/propidium iodide (PI) staining. Cell death was determined using flow cytometry. The INS-1 cells were stained with Annexin V and PI using the Annexin-V-FLUOS staining kit (Roche Diagnostics, Basel, Switzerland) in accordance with the manufacturer's protocol. Briefly, the cells were plated in 24-well plates at a density of $1.2 \times 10^{5}$ cells per $\mathrm{cm}^{2}$, treated with lysosomal proteases and stained with an incubation buffer containing Annexin V and PI for 10 min at $15-25^{\circ} \mathrm{C}$ in the dark. Images of the apoptotic cells were captured using a fluorescence microscope (Nikon Eclipse TE2000-U; Nikon Corporation, Tokyo, Japan) with an excitation wavelength in the range of 450-500 $\mathrm{nm}$ and detection in the range of 515-565 nm (12).

Western blot analysis. Cells were washed with PBS and lysed in lysis buffer [62.5 mM Tris- $\mathrm{HCl}$ (pH 6.8), $2 \%$ SDS, 5\% BME, 1\% TritonX-100, 1 mM EDTA, 1 mM EGTA, $10 \mathrm{mM}$ DTT and $\left.1 \mathrm{~mm} \mathrm{Na} \mathrm{VO}_{4}\right)$. The lysates were then incubated on ice for $30 \mathrm{~min}$ and centrifuged at $8,000 \mathrm{x}$ g for $5 \mathrm{~min}$ at $4^{\circ} \mathrm{C}$. Protein concentrations were determined using the Bicinchoninic Acid method. Equal quantities of protein $(50 \mu \mathrm{g})$ were resolved by $10-15 \%$ SDS-PAGE, transferred onto polyvinylidine difluoride membranes and blocked with $5 \%$ skim milk at room temperature for $1 \mathrm{~h}$. The membranes were incubated with specific primary antibodies overnight at $4^{\circ} \mathrm{C}$. The following antibodies were used at a 1:1,000 dilution (unless otherwise indicated): LC3 (Cell Signaling Technology, Inc., Danvers, MA, USA; cat. no. 2775), IL1 $\beta$ (Cell Signaling Technology, Inc.; cat. no. 2002), caspase-1 (Abcam, Cambridge, UK; cat. no. 2225), C/EBP homologous protein (CHOP; Cell Signaling
Technology, Inc.; cat. no. 2895) and $\beta$-actin (Cell Signaling Technology, Inc.; cat. no. 8475). The membranes were washed with PBS-0.1\% Tween-20, then incubated with horseradish peroxidase-conjugated secondary antibodies (1:5,000; Sigma; Merck Millipore) at room temperature for $1 \mathrm{~h}$ (10). Protein bands were detected using Enhanced chemiluminescence (Beyotime Institute of Biochemistry, Haimen, China). Immunoblots were quantified by densitometric analysis using Image Lab software v2.0.1 (Bio-Rad Laboratories, Inc., Hercules, CA, USA). The quantification of protein phosphorylation was normalized to the corresponding total protein expression. The relative expression level of a certain protein was normalized to $\beta$-actin and experiments were repeated in triplicate.

RNA analysis. To analyze mRNA expression, total RNA was extracted using TRNzol reagent (Tiangen Biotech Co., Ltd., Beijing, China; cat. no. DP405-02) according to the manufacturer's instructions. The purity and integrity of the RNA were examined spectroscopically prior to obtaining cDNA using the Nanodrop 2000. Reverse transcription was performed using the PrimeScript ${ }^{\mathrm{TM}}$ RT reagent kit with gDNA Eraser system (Takara Bio, Inc., Otsu, Japan; cat. no. RR047B) according to the manufacturer's instructions. The reactions were set up with the following master mix: $2.0 \mu \mathrm{l}$ gDNA Eraser Buffer, $1.0 \mu \mathrm{l}$ gDNA Eraser, $1 \mu \mathrm{g}$ Total RNA and $6.0 \mu \mathrm{l}$ RNase Free $\mathrm{H}_{2} \mathrm{O}$, which was incubated for $2 \mathrm{~min}$ at $42^{\circ} \mathrm{C}$. This master mix was then mixed with $1.0 \mu \mathrm{l}$ PrimeScript RT Enzyme Mix I, $1.0 \mu \mathrm{l}$ RT Primer Mix, $4.0 \mu$ l PrimeScript Buffer 2 and $4.0 \mu \mathrm{l}$ RNase Free $\mathrm{H}_{2} \mathrm{O}$, and incubated at $37^{\circ} \mathrm{C}$ for $15 \mathrm{~min}$ then at $85^{\circ} \mathrm{C}$ for $5 \mathrm{sec}$. For thermocycling reactions, the $\mathrm{ABI}$ prism 7500 sequencer detection system was used. Using the SYBR ${ }^{\circledR}$ Premix Ex Taq ${ }^{\mathrm{TM}}$ II (Tli RNaseH Plus) and the ROX plus reaction system (both Takara Bio, Inc.; cat. no. RR82LR), the following thermocycling conditions were applied: $95^{\circ} \mathrm{C}$ for $30 \mathrm{sec}$, then 45 cycles of $95^{\circ} \mathrm{C}$ for $5 \mathrm{sec}$ and $60^{\circ} \mathrm{C}$ for $40 \mathrm{sec}$. The relative quantity of each transcript was calculated by a standard curve of quantification cycle values for serial dilutions of a cDNA sample. PCR was performed in triplicate for each sample. The following sets of primers were used for PCR analysis: Rat actin forward 5'-GGA GATTACTGCCCTGGCTCCTA-3' and reverse 5'-GACTCA TCGTACTCCTGCTTGCTG-3'; rat binding immunoglobulin heavy chain protein (Bip), forward 5'-GAATCCCTCCTGCTC CCCGT-3' and reverse 5'-TTGGTCATTGGTGATGGTGAT TTTG-3', rat activating transcription factor 4 (ATF4), forward 5'-TATGAGCCCTGAGTCCTACCTG-3' and 5'-CTGCTG TCTTGTTTTGCTCCAT-3'. The primers used for analysis were designed and synthesized by Invitrogen; Thermo Fisher Scientific, Inc. (Waltham, MA, USA). All experiments were performed in triplicate and the relative mRNA levels were analyzed using the $2^{-\Delta \Delta \mathrm{Cq}}$ method (13).

Statistical analysis. The results are expressed as the mean \pm standard error of the mean. Comparisons of a single variable in $>2$ groups were analyzed using one-way analysis of variance followed by Tukey's multiple comparison tests using GraphPad Prism (GraphPad Software, Inc., La Jolla, CA, USA). Statistical analysis was performed using the paired and unpaired t-test between two groups using SPSS 12.0 software (SPSS, Inc., Chicago, IL, USA). P<0.05 was considered to indicate a statistically significant difference. 
A

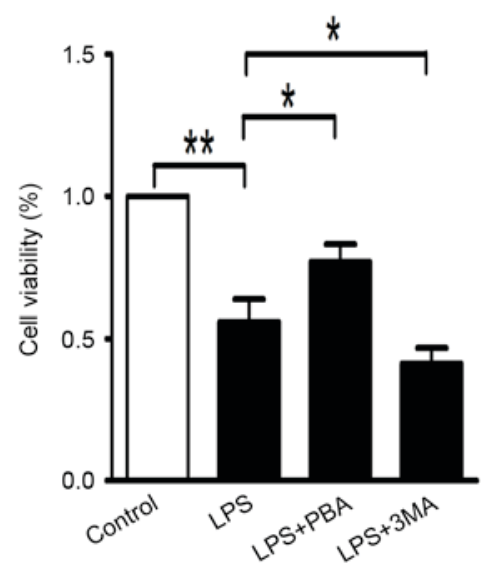

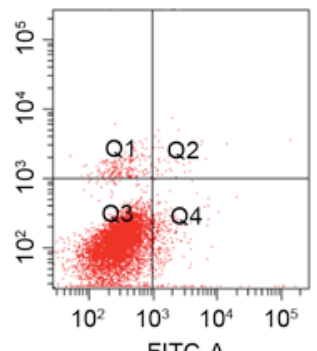

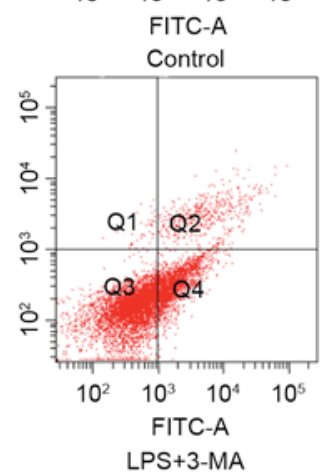

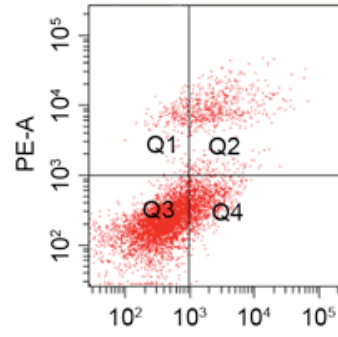

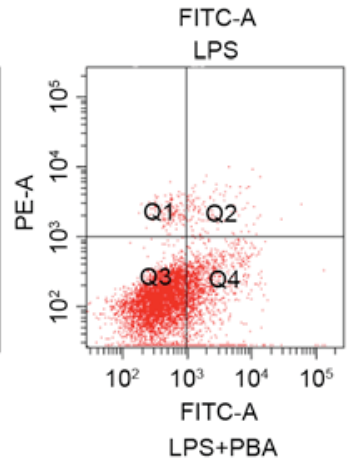

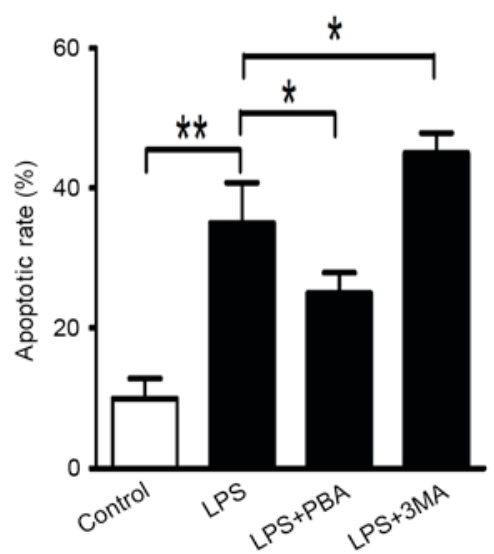

Figure 1. Detection of the survival status of INS-1 cells treated with LPS and inhibitor of endoplasmic reticulum stress/autophagy. INS-1 cells were incubated with LPS $100 \mathrm{ng} / \mathrm{ml}$ for $24 \mathrm{~h}$ to simulate the conditions of diabetes. INS-1 cells were treated with a combination of LPS and PBA (5 mmol/1)/3-MA ( $5 \mathrm{mmol} / \mathrm{l}$ ) for $24 \mathrm{~h}$ as the LPS+PBA group and the LPS+3-MA group, respectively. (A) Relative cell viability was determined using a Cell Counting kit-8 assay. (B) Apoptotic rates were determined using flow cytometry. Values are presented as the mean \pm standard deviation $\left(\mathrm{n}=3\right.$ ). ${ }^{*} \mathrm{P}<0.05$, ${ }^{* *} \mathrm{P}<0.01$. LPS, lipopolysaccharide; PBA, 4-phenylbutyric acid; 3-MA, 3-methyadenine.

A

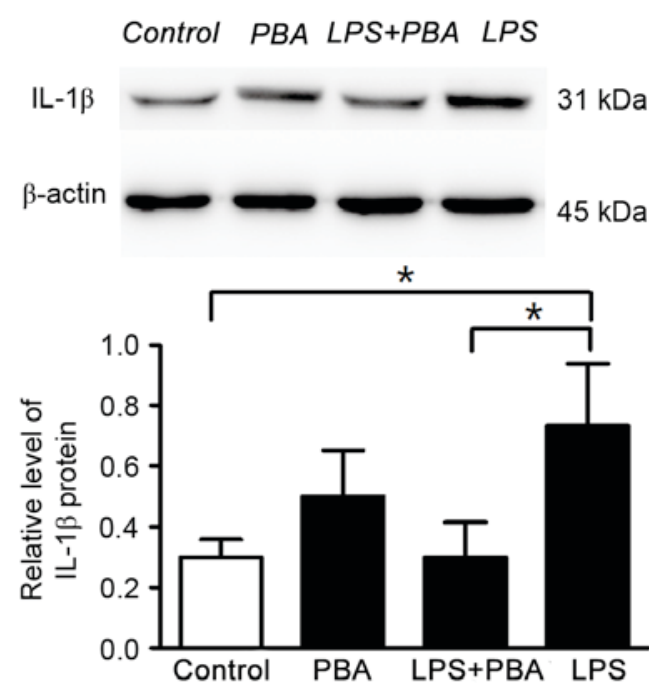

B
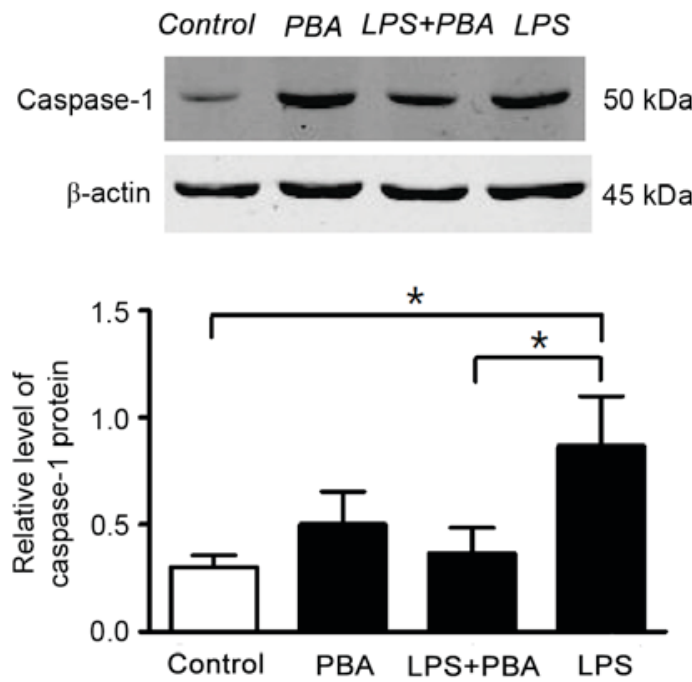

Figure 2. Detection of protein expression levels of mature caspase-1 and inflammatory cytokines IL-1 $\beta$ in INS-1 cells treated them LPS or LPS and the inhibitor of endoplasmic reticulum stress. (A) Protein levels of IL-1 $\beta$ and (B) caspase-1 were normalized to $\beta$-actin. Values are presented as the mean \pm standard deviation $(\mathrm{n}=3)$. $\mathrm{P}<0.05$. LPS, lipopolysaccharide; PBA, 4-phenylbutyric acid; IL-1 $\beta$, interleukin-1 $\beta$.

\section{Results}

LPS induces the production of IL1 $\beta$, inflammasome activation and apoptosis in INS-1 cells. The INS-1 cells were incubated with $100 \mathrm{ng} / \mathrm{ml}$ LPS in RPMI-1640 for $24 \mathrm{~h}$. As shown in Fig. 1A, the viability of the INS-1 cells analyzed using the CCK8 assay was reduced to $64 \%$ with LPS treatment, compared with that in the control group. Apoptosis of cells in the LPS group was increased by $26.8 \%$, determined using an Annexin V-FITC/PI quantification assay (Fig. 1B).
PBA attenuates LPS-induced production of ILI $\beta$ and enhances survival of in INS-1 cells. The INS-1 cells were treated with LPS and PBA. PBA is a low-molecular-weight compound, which is known to reduce the load of unfolded proteins in the ER by facilitating folding capacity and trafficking of mutant proteins out of the ER. Ozcan et al (14) and Tang et al (15) demonstrated that PBA decreases the activation of IRE-1, but not of the PERK or ATF6 pathways. On the basis of previous experimental analysis, the present study used PBA as a chemical chaperone to inhibit IRE-1 pathway-mediated 
ER stress. The survival of INS-1 cells was improved to $78 \%$ (Fig. 1A), and the percentage of apoptotic cells was reduced to $21.2 \%$ (Fig. 1B) following treatment with LPS and PBA ( $5 \mathrm{mmol} / \mathrm{l}$ ) for $24 \mathrm{~h}$. In the LPS+PBA-treated INS-1 cells, the protein expression levels of IL-1 $\beta$ (Fig. 2A) and caspase-1 (Fig. 2B) were reduced, compared with those in the groups treated with LPS alone. These results suggested that the modulation of ER stress reduced the production of inflammatory cytokine (IL-1 $\beta$ and improved INS-1 cell survival.

IRE-1-pathway-mediated ER stress is involved in the process of inflammation and autophagy. As LC3B-II is a key protein associated with autophagy, the conversion of LC3B-I to LC3B-II was examined in the present study. There were fewer autophagic vacuoles (AVs; Fig. 3A, blue arrowheads), and a number of swelling mitochondria (Fig. 3A, green arrowheads) detected in the LPS+PBA group, determined using EM. In addition, the LPS-induced LC3B-II protein levels were reduced in the LPS+PBA group, compared with those in the LPS-treated group (Fig. 3B). These results suggested that IRE-1-pathway-mediated ER stress was involved in the association between inflammation and autophagy, and in promoting the processes of inflammation and autophagy.

Autophagy decreases INS-1 cell death induced by the inflammatory response. To determine the role of autophagy in the inflammatory response, the INS-1 cells were treated with autophagy inhibitor (3-MA). INS-1 cell viability was significantly reduced to $42 \%$ by treatment with LPS $+3-$ MA (Fig. 1A). 3-MA increased the apoptotic rate to $43.8 \%$ in the LPS+3-MA group (Fig. 1B). Additionally, the typical autophagosome (Fig. 3A, red arrowhead) double-limiting membrane in the LPS-treated INS-1 cells was detected by EM. Double-membrane autophagic vesicles containing cell organelles in the cytoplasm of INS-1 cells is an integrated autophagosome, shown by ultrastructural image analysis. There were numerous different periods of autophagosomes (Fig. 3A, red arrowheads) and autophagolysosomes (ALs) in the LPS group. These results indicated that autophagy had a protective effect on the LPS-induced inflammatory response in INS-1 cells and was necessary to maintain the normal architecture and function of INS-1 cells. The mRNA expression levels of Bip and ATF4, and the protein expression of CHOP were assessed following treatment with 3-MA. The mRNA levels of Bip and ATF4, and the protein expression of CHOP were modestly upregulated (Fig. 4A-C). These data suggested that autophagy protected INS-1 cells by attenuating excessive ER stress, and decreasing cell apoptosis mediated by PERK/CHOP-mediated ER-stress and the production of inflammatory cytokines (IL1 $\beta$ ).

\section{Discussion}

Type 2 diabetes is closely associated with the activation of inflammatory signaling pathways, resulting in a marked increase in autophagy, in addition to abnormal cytokine production (16). These results suggested that pancreatic $\beta$ cells can secrete cytokine IL1 $\beta$, which is involved in the inflammatory response and is detrimental to cell survival. Autophagy is also involved in type 2 diabetes, however, whether autophagy promotes INS-1 cell death or protects cells from injuries remains to be elucidated. Therefore, INS-1 cells were treated with autophagy inhibitor (3-MA). The further decrease in proliferation and increased apoptosis of INS-1 cells suggested that autophagy protected the cells from death.

Type 2 diabetes is associated with insulin resistance, cell depletion, and immune attack, involving lipotoxicity, glucotoxicity and inflammation (17). Several studies have demonstrated that the intra-islet expression of inflammatory cytokines, particularly IL-1 $\beta$, contributes to the pathogenesis of type 2 diabetes. The most prominent feature of type 2 diabetes, compared with type 1 diabetes, is that the islet inflammatory response is 'low-grade' and its role in the pathophysiology of type 2 diabetes is somewhat controversial (18). In the present study, pancreatic $\beta$ cells secreted cytokines, including IL1 $\beta$, which was involved in the inflammatory response and is detrimental to the survival of $\beta$-cells. These results are consistent with a previous study by Böni-Schnetzler et al (19). This previous study differed in that the RNA from $\beta$-cells of individuals with type 2 diabetes were analyzed, and it was revealed that the mRNA expression of IL1 $\beta$ was induced by high glucose and IL1 $\beta$ autostimulation, and was decreased by the IL-1 receptor antagonist IL-1Ra. Therefore, the production of inflammatory cytokines may be a positive feedback mechanism, and the autostimulation of IL1 $\beta$ is transient and nuclear factor- $\kappa \mathrm{B}$-dependent (19).

Clinical and experimental evidence suggests that ER stress is a potent, evolutionarily conserved response to misfolded proteins and cellular metabolic stress, which contributes to the life-and-death decisions of $\beta$-cells during type 2 diabetes. The inflammatory response is frequently triggered as a consequence of ER stress, caused either by metabolic problems or by the accumulation of misfolded proteins (20). Oslowski et al (4) showed that TXNIP is a critical signaling node, which links ER stress to inflammation. TXNIP induced by ER stress is under the control of PERK and IRE1 pathways, which induce the mRNA transcription of IL1 $\beta$ (4). IL1 $\beta$ is activated by the NLRP3 inflammasome and mediates ER stress-mediated $\beta$-cell death. Activation induces oligomerisation of the NLRP3 inflammasome and recruits ASC through a homotypic PYD-PYD interaction. The ASC then recruits pro-caspase-1, leading to the autocatalytic activation of caspase-1, and these active caspase- 1 hetero-tetramers can convert inactive pro-IL1 $\beta$ into their bioactive and secreted forms (3). The results of the present study demonstrated that modulating ER and increase folding capacity can reduce inflammatory cytokines (IL1 $\beta$ ) and improve INS-1 cell survival. The results also suggested that IRE-1-pathway-mediated ER stress is a key molecule linking inflammation to autophagy, which may contribute to the death and dysfunction of $\beta$-cells, caused by a combination of glucotoxicity and lipotoxicity (21).

Autophagy is an evolutionary conserved process, which has two effects on cell survival. The data presented in the present study suggested that autophagy is a cell-protection mechanism. However, Eskelinen and Saftig suggested that, when the disposal mechanism is carried to excess, the cells are committed to die, dependent on autophagy (22). In mammalian cells, autophagy is activated by ER stress, with IRE1-JNK as a link between the two processes, and the kinase function 
A
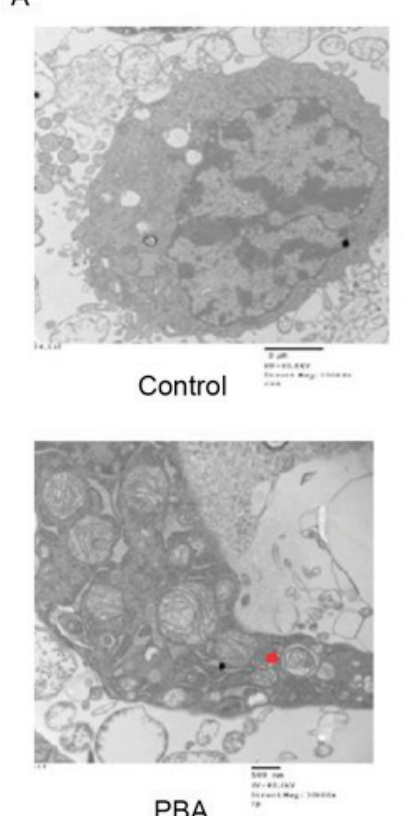
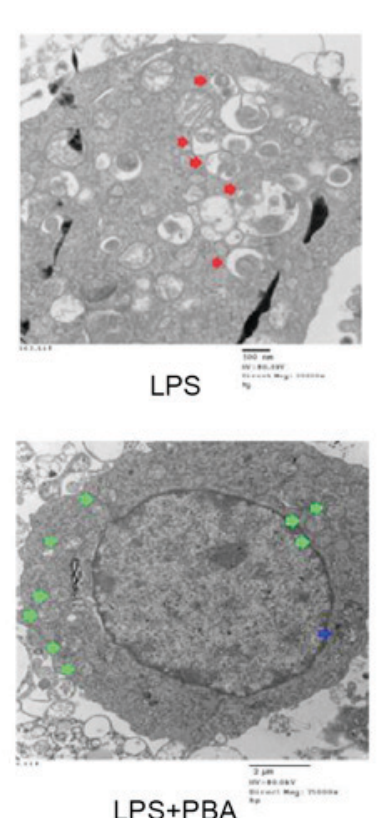

B
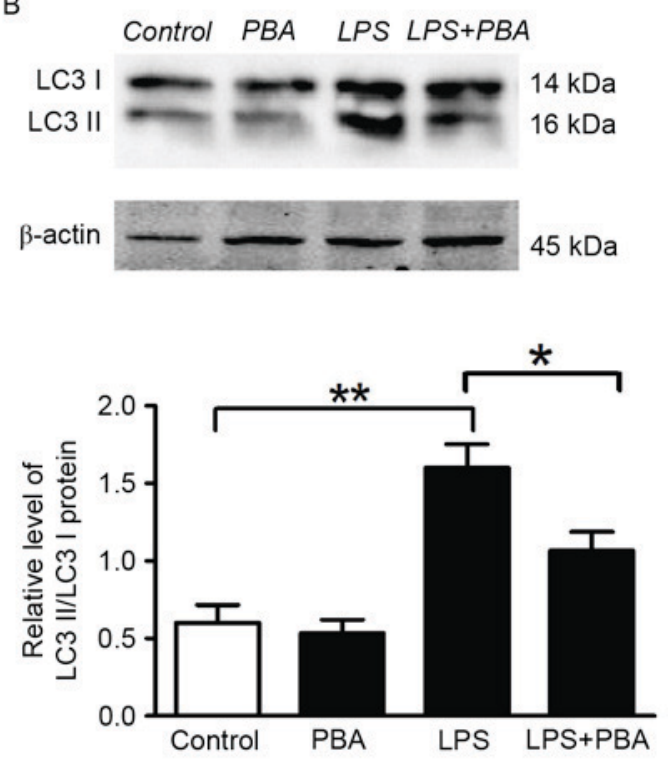

Figure 3. (A) Micromorphological changes in cellular organelles examined by transmission electron microscopy; autophagosomes (red arrowheads), autophagic vacuoles (blue arrowheads) and swelling mitochondria (green arrowheads) were observed. (B) Western blot analysis was used to detect the expression of LC3 in INS-1 cells. Relative expression of LC 3 was normalized to $\beta$-actin. Data are expressed as the mean \pm standard deviation ( $n=3$ for each group). "P $<0.05$, ${ }^{* *} \mathrm{P}<0.01$. LPS, lipopolysaccharide; PBA, 4-phenylbutyric acid; LC3, light chain 3.

A

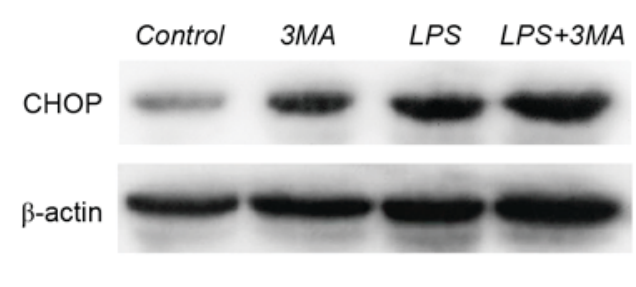

B

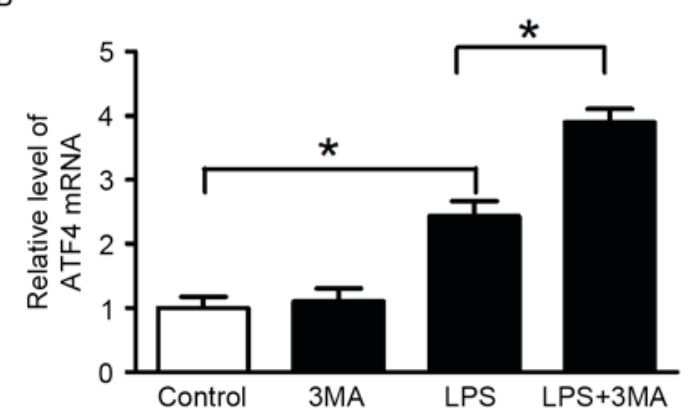

$27 \mathrm{kDa}$

$45 \mathrm{kDa}$

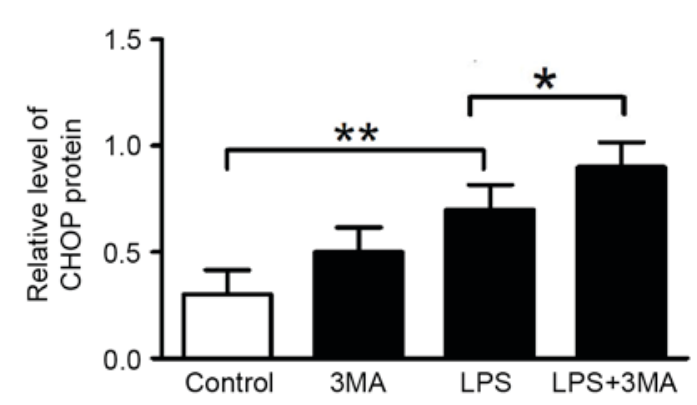

C

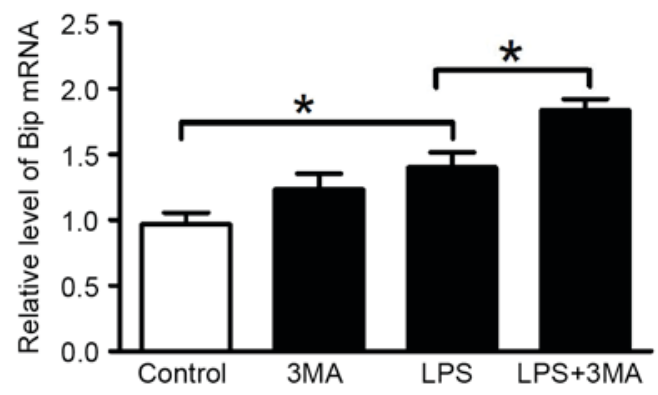

Figure 4. (A) Protein expression of CHOP; (B) mRNA expression of ATF4; (C) mRNA expression of Bip. Data are expressed as the mean \pm standard error of the mean ( $\mathrm{n}=3$ for each group). ${ }^{\mathrm{P}} \mathrm{P}<0.05,{ }^{* *} \mathrm{P}<0.01$, compared with the control. LPS, lipopolysaccharide; PBA, 4-phenylbutyric acid; 3-MA, 3-methyadenine; CHOP, C/EBP homologous protein; ATF4; activating transcription factor 4; Bip, binding immunoglobulin heavy chain protein.

of IRE1 is required for the induction of autophagy. However, PERK and ATF6 pathways are not considered to be important in the activation of autophagy following ER stress (7). In the present study, inhibiting IRE-1 mediated ER stress not only through reducing the protein expression of caspase-1 and IL1 $\beta$, but also by depressing the LC3-II/LC3-I ratio, compared with levels in the LPS-treated group. There are two possible reasons for these results. It may be that autophagy, which is induced by inflammatory cytokines, is reduced, followed by decreased expression levels of caspase- 1 and IL $1 \beta$. Alternatively, it may be that the autophagy induced by IRE-1-mediated ER stress is decreased. However, the production of inflammatory cytokines (IL1 $\beta$ ) is also mediated by the IRE-1 pathway. Therefore, the results of the present study suggested that IRE1 is a link 
between autophagy and ER stress in INS-1 cells. Additionally, Gonzalez et al (6) suggested that LC3I can be converted to the lipidated form (LC3-II), mediated by the phosphorylation of PERK/eIF2 $\alpha$, and the connection between autophagy and ER stress requires further investigation.

Inflammasome and autophagy are essential elements of the innate immune system, and their disruption has been implicated in the pathogenesis of type 2 diabetes. ER has previously been tightly linked to autophagy and inflammation, and has been considered as an intersection integrating multiple stress responses, and these processes are associated with the pathogenesis of diabetes mellitus and its complications. The results of the present study showed that the IRE1-mediated ER stress pathway activated the production of IL1 $\beta$. Autophagy was also activated by the IRE1 pathway, which suggested that IRE1-mediated ER stress is essential for inflammatory cytokine secretion and the activation of autophagy. The results of the present study and previous findings, which link autophagy and inflammasome regulation, suggest that autophagy cooperates with inflammation and apoptosis, and the adaptive immune system, to orchestrate cellular homeostasis against danger signals, including ER stress. The data obtained in the present study, when combined with previous findings, indicate that there is a tight link between ER stress and inflammation autophagy, suggesting that a therapeutic strategy, which aims to target the common molecular processes altered in $\beta$-cells, may be effective.

In conclusion, the present study indicated that LPS induced am NLRP3-dependent proinflammatory response via excessive ER stress. Autophagy was shown to be important in ameliorating ER stress and NLRP3-dependent inflammatory cytokine secretion, further reducing INS-1 cell death. It was also shown that IRE-1-pathway-mediated ER stress is a crucial link connecting autophagy with the NLRP3-dependent inflammatory response.

\section{Acknowledgements}

The present study was supported by the National Natural Science Foundation of China (grant no. 81370929) and China Diabetes Young Scientific Talent Research Funding (2016).

\section{References}

1. Eizirik DL and Mandrup-Poulsen T: A choice of death-the signal-transduction of immune-mediated beta-cell apoptosis. Diabetologia 44: 2115-2133, 2001.

2. Kaminitz A, Stein J, Yaniv I and Askenasy N: The vicious cycle of apoptotic beta-cell death in type 1 diabetes. Immunol Cell Biol 85: 582-589, 2007.

3. Donath MY and Shoelson SE: Type 2 diabetes as an inflammatory disease. Nat Rev Immunol 11: 98-107, 2011.

4. Oslowski CM, Hara T, O'Sullivan-Murphy B, Kanekura K, Lu S, Hara M, Ishigaki S, Zhu LJ, Hayashi E, Hui ST, et al: Thioredoxin-interacting protein mediates ER stress-induced $\beta$ cell death through initiation of the inflammasome. Cell Metab 16: 265-273, 2012 .
5. Rabinowitz JD and White E: Autophagy and metabolism. Science 330: 1344-1348, 2010.

6. Gonzalez CD, Lee MS, Marchetti P, Pietropaolo M, Towns R, Vaccaro MI, Watada H and Wiley JW: The emerging role of autophagyin the pathophysiology of diabetes mellitus. Autophagy 7: 2-11, 2011.

7. Ogata M, Hino S, Saito A, Morikawa K, Kondo S, Kanemoto S, Murakami T, Taniguchi M, Tanii I, Yoshinaga K, et al: Autophagy is activated for cell survival after endoplasmic reticulum stress. Mol Cell Biol 26: 9220-9231, 2006.

8. Liu Y, Burgos JS, Deng Y, Srivastava R, Howell SH and Bassham DC: Degradation of the endoplasmic reticulum by autophagy during endoplasmic reticulum stress in Arabidopsis. Plant Cell 24: 4635-4651, 2012.

9. Yang X, Srivastava R, Howell SH and Bassham DC: Activation of autophagy by unfolded proteins during endoplasmic reticulum stress. Plant J 85: 83-95, 2016.

10. Jing Yin J, Bo Li Y, Ming Cao M and Wang Y: Liraglutide improves the survival of INS-1 cells by promoting macroautophagy. Int J Endocrinol. Metab 11: 184-190, 2013.

11. Akerfeldt MC, Howes J, Chan JY, Stevens VA, Boubenna N, McGuire HM, King C, Biden TJ and Laybutt DR: Cytokine-induced beta-cell death is independent of endoplasmic reticulum stress signaling. Diabetes 57: 3034-3044, 2008.

12. Chan JY, Cooney GJ, Biden TJ and Laybutt DR: Differential regulation of adaptive and apoptotic unfolded protein response signalling by cytokine-induced nitric oxide production in mouse pancreatic beta cells. Diabetologia 54: 1766-1776, 2011.

13. Livak KJ and Schmittgen TD: Analysis of relative gene expression data using real-time quantitative PCR and the 2(-Delta Delta C(T)) method. Methods 25: 402-408, 2001.

14. Ozcan U, Yilmaz E, Ozcan L, Furuhashi M, Vaillancourt E, Smith RO, Görgün CZ and Hotamisligil GS: Chemical chaperones reduce ER stress and restore glucose homeostasis in a mouse model of type 2 diabetes. Science 313: 1137-1140, 2006.

15. Tang C, Koulajian K, Schuiki I, Zhang L, Desai T, Ivovic A, Wang P, Robson-Doucette C, Wheeler MB, Minassian B, et al: Glucose-induced beta cell dysfunction in vivo in rats: Link between oxidative stress and endoplasmic reticulum stress. Diabetologia 55: 1366-1379, 2012.

16. Kosacka J, Kern M, Klöting N, Paeschke S, Rudich A, Haim Y, Gericke M, Serke H, Stumvoll M, Bechmann I, et al: Autophagy in adipose tissue of patients with obesity and type 2 diabetes. Mol Cell Endocrinol 409: 21-32, 2015.

17. Liu H, Cao MM, Wang Y, Li LC, Zhu LB, Xie GY and Li YB: Endoplasmic reticulum stress is involved in the connection between inflammation and autophagy in type 2 diabetes. Gen Comp Endocrinol 210: 124-129, 2015.

18. Imai Y, Dobrian AD, Morris MA and Nadler JL: Islet inflammation: A unifying target for diabetes treatment? Trends Endocrinol Metab 24: 351-360, 2013.

19. Böni-Schnetzler M, Thorne J, Parnaud G, Marselli L, Ehses JA, Kerr-Conte J, Pattou F, Halban PA, Weir GC and Donath MY: Increased interleukin (IL)-1beta messenger ribonucleic acid expression in beta -cells of individuals with type 2 diabetes and regulation of IL-1beta in human islets by glucose and autostimulation. J Clin Endocrinol Metab 93: 4065-4074, 2008.

20. Hotamisligil GS: Endoplasmic reticulum stress and the inflammatory basis of metabolic disease. Cell 140: 900-917, 2010.

21. Quan W, Jo EK and Lee MS: Role of pancreatic $\beta$-cell death and inflammation in diabetes. Diabetes Obes Metab 3: 141-151, 2013.

22. Eskelinen EL and Saftig P: Autophagy: A lysosomal degradation pathway with a central role in health and disease. Biochim Biophys Acta 1793: 664-673, 2009. 